\title{
Serviço Social e desastres: campo para o conhecimento e a atuação profissional
}

\author{
Social Service and disasters: \\ field for knowledge and the professional performance
}

\begin{abstract}
Maria Roseli Rossi Avila
Assistente social, Universidade Regional de Blumenau, doutoranda no Programa de Pós-Graduação em Desenvolvimento Regional, bolsista Capes. Blumenau/Santa Catarina, Brasil.

mariaroselirossiavila@yahoo.com.br
\end{abstract}

Marcos Antonio Mattedi

Sociólogo, doutor em Sociologia, Universidade Regional de Blumenau, docente no Programa de Pós-Graduação em Desenvolvimento Regional, Blumenau/SC, Brasil.

mattediblu@gmail.com

Maria Salete da Silva

Assistente social, doutora em Sociologia, Universidade Regional de Blumenau, docente no Departamento de Serviço Social, Blumenau/SC, Brasil.

mssilva@fubr.br

Resumo: O artigo aborda o tema dos desastres e Serviço Social. Visa contribuir para a inclusão da temática na agenda de pesquisa e intervenção da profissão. Argumenta que o Serviço Social tem uma postura central na gestão das vulnerabilidades a desastres, mas discute pouco a questão. Para isso, apresenta resultados de pesquisa sobre o debate teórico e a produção recente do Serviço Social na área dos desastres no Brasil.

Palavras-chave: Serviço Social. Desastres. Vulnerabilidade. Gestão. Agenda social.
Abstract: The article approaches the issue of disasters and Social Service. It aims at including such an issue in the research and intervention agenda of the profession. It reasons that although Social Service has a central posture in the management of vulnerabilities and disasters, it does not discuss the issue enough. In order to do that, the article presents research results about the theoretical debate and Social Service recent production related to disasters in Brazil.

Keywords: Social Service. Disasters. Vulnerability. Management. Social agenda. 


\section{Introdução}

or décadas os desastres foram considerados somente a partir de suas
características geofísicas e, portanto, objeto de estudo das ciências natu-
rais e das engenharias. Após serem considerados fenômenos sociais (Perry e Quarantelli, 2005), passaram a ser estudados pela sociologia e as ciências humanas em geral. Porém há uma lacuna no debate sobre os desastres no Serviço Social. Como campo de pesquisa e atuação profissional, o desenvolvimento de estudos sobre as questões ambientais é recente. Somente a partir da década de 1990 a categoria passou a discutir o meio ambiente e sua relação com a profissão (Loureiro, 2010; Nunes, 2013). E bem mais recente é a inserção dos desastres socioambientais nos debates da categoria, embora a centralidade da profissão na gestão da crise (pós-impacto dos desastres) já ocorra há mais tempo.

A problemática dos desastres globalizou-se, inseriu-se e passou a orientar a agenda social internacional e as ações na área (Phillips et al., 2010; Revet, 2011). Diversos autores realizaram pesquisas que resultaram em aportes teóricos consistentes que orientam as discussões sobre a temática até os dias de hoje. Os resultados trouxeram novas e substanciais contribuições para a implantação de políticas públicas na área e para a gestão dos desastres no mundo. A Organização das Nações Unidas (ONU), objetivando reduzir o impacto dos desastres no mundo, designou, em 1990, a Década Internacional para Redução de Desastres (International Decade for Natural Disaster Reduction - IDNDR). A partir de lá, foram realizadas três conferências mundiais (UNISDR, 2015), as de Yokohama (1994), de Hyogo (2005) e de Sendai (2015). Esta última mudou o marco referencial adotado até então de gestão dos desastres (GD) para gestão do risco de desastres (GRD).

Apesar das tentativas para a redução de desastres, dados do EM-DAT ${ }^{1}$ (D. Guha-Sapir, Below e Hoyois, 2015) apontam que os eventos aumentaram de oitenta por ano (1970) para quatrocentos (2008). Também os prejuízos passaram de 5 bilhões de dólares em 1975 para 180 bilhões em 2008 e 300 bilhões

1. A EM-DAT é uma base de dados com registro de mais de 18 mil grandes desastres ocorridos em todo o mundo desde 1900 (Freiria, 2011). 
em 2015. No entanto, apesar desses aumentos, o número de mortes diminuiu significativamente (ONU, 2015). O Brasil segue a tendência mundial. Entre 1990 e 2012 foram registradas 38.996 ocorrências no país. Deste montante, 8.515 aconteceram na década de 1990 , representando $22 \%$, enquanto 21.741 foram na década de 2000, totalizando 56\%. Já nos anos 2010, 2011 e 2012 foram 8.740 eventos, $22 \%$ do total. Isso significa que em apenas três anos tivemos um aumento de 40\% no número de ocorrências (Ceped, 2013).

A densidade populacional relacionada à pobreza também influencia no impacto dos desastres. $\mathrm{O}$ crescimento das cidades tem sido acompanhado pelo crescimento desproporcional da pobreza. E as populações mais empobrecidas quase sempre estão localizadas em áreas ecologicamente vulneráveis, como planícies inundáveis, encostas íngremes e moradias com infraestrutura precária (Aysan, 1999). Quanto maior a pobreza e a densidade populacional da região atingida pelo desastre, maior a vulnerabilidade, maior o impacto e as perdas decorrentes. Portanto, é fundamental a inserção do debate sobre vulnerabilidade e gestão dos desastres na agenda do Serviço Social. A relação entre Serviço Social e desastres está, muitas vezes, restrita ao campo da intervenção dos(as) assistentes sociais junto à população atingida pelos eventos (Samagaia e Angioni, 2009; Santos, 2012; Nunes e Silva, 2013).

A profissão tem uma larga história na gestão dos desastres e no atendimento da população em momentos de crise. A atuação do(a) assistente social permite que a população atingida tenha acesso aos serviços básicos disponíveis e seus direitos fundamentais garantidos durante e após a crise (Zakour, 2007 e 2008). Para Zakour (2007), o Serviço Social atua na prestação de serviços que oferecem alívio e mitigação da crise à população atingida e o necessário à sobrevivência em meio à situação de emergência gerada pelo desastre. Contudo, a produção teórica e as estratégias de intervenção social são incipientes na área (Nunes e Silva, 2013). Conforme Zakour (2007), a intervenção social que hoje incide sobre a mitigação, preparação, resposta e períodos de recuperação de desastres deve avançar para o campo da formação, da investigação teórica e da produção científica.

Portanto, este estudo tem como objetivo contribuir para o debate a respeito da inclusão dos desastres na agenda do Serviço Social, como campo de 
pesquisa, formação e intervenção profissional. Além da "Introdução" e das "Considerações finais", dividimos o texto em três partes. Na primeira, apresentamos uma abordagem dos desastres, os primeiros grupos de pesquisa, as revistas especializadas na área, os comitês e as associações científicas. Na segunda, discutimos a gestão dos desastres, as relações pré e pós-impacto dos eventos e apresentamos uma proposta de Modelo Multidimensional de Gestão de Risco de Desastres. E na terceira tratamos do Serviço Social e desastres. Apresentamos resultados de pesquisas que comprovam a incipiência da discussão na área e a necessidade da inserção da temática dos desastres na agenda social da profissão.

\section{A abordagem dos desastres}

As pesquisas sobre os desastres tiveram início, no campo da sociologia, em 1920. Desde lá, inúmeras perspectivas de estudo foram desenvolvidas, a produção do conhecimento entrou para a agenda governamental, foi disseminada e globalizada, dando origem a vários grupos de pesquisa, entre eles o Disasters Center Research - University of Delaware - e o Centre for Research on the Edpidemiology in Disasters - EM-DAT. Inúmeras revistas, como Environmental Hazards, Risk Analysis e Natural Hazards Review, além de vários comitês científicos, especializaram-se na temática. As iniciativas visando a redução do risco de desastres estão ligadas ao Bulding a Culture of Prevention e à Declaração do Decênio Internacional para Redução de Desastres Naturais (1990-2000). As mesmas se estendem para as conferências sobre disasters risk redution e para os marcos de Yokohama, Hyogo e Sendai (UNISDR, 2015).

No entanto, quanto mais informações sobre a problemática, maiores as polêmicas. Segundo Perry e Quarantelli (2005), mesmo após tanto tempo de pesquisas não há total concordância sobre o que é um desastre. Disputas conceituais entre disciplinas da área social e técnica constituem um desafio tanto no plano cognitivo como no político. No cognitivo, a disputa inclui discussões a respeito do paradigma da ameaça externa e do paradigma da vulnerabilidade interna. E, no político, situa-se no âmbito da gestão. Mais especificamente, a oposição entre as medidas de preparação e de recuperação, e entre a centralização estatal e a organização comunitária. Para entender esses dilemas, faz-se 
necessário conhecer a origem dos estudos sobre os desastres, iniciada com S. H. Prince (1920), que pesquisou a explosão entre dois navios no porto de Halifax (Canadá), em 1915, e concluiu que a mesma não causou danos ao município (Prince, 1920; Marchezini, 2010).

Só após a Segunda Guerra Mundial, o campo de estudos se estabeleceu, destacando-se duas formas de abordagens: a geográfica e a sociológica (Quarantelli, 1978). A abordagem geográfica considera os fatores naturais dos desastres, e as pesquisas remontam a Gilbert White (1945), que estudou enchentes nos Estados Unidos (Kates, 2011). Até então, os estudos visavam reduzir o impacto das enchentes com obras estruturais (de melhoramento e retenção fluvial). Para White, essas medidas não diminuíam os danos, por isso inseriu a variável humana (ajustamento humano) às enchentes e iniciou estudos sobre terremotos, erupções vulcânicas, tornados, furacões, secas etc. A inclusão originou o campo de estudo dos hazards, definidos "como elementos do ambiente físico prejudiciais para o homem, os quais surgem do contínuo processo de ajustamento entre sistema humano e eventos naturais" (Mattedi e Butzke, 2001, p. 4).

Outro estudioso dos hazards (da ecologia humana) foi Kenneth Hewitt (2010). Hewitt preocupou-se com o campo do risco e dos desastres a partir de três perspectivas: do perigo (ameaça — hazard), da vulnerabilidade e da resposta. Para Hewitt (1997), a preparação pré-evento poderia melhorar as possibilidades de sobrevivência e recuperação das regiões no pós-evento. Assim, a partir de 1970, a variável humana foi incorporada às forças físicas dos hazards para determinar a magnitude e o impacto do evento. Eventos cuja dimensão física não afeta as atividades humanas não são considerados hazards. Essa mudança na abordagem (sociológica) dos hazards (ajustamento humano + disrupção provocada pelo agente físico) enfatiza a "percepção dos hazards, ajustamento e escolha de respostas, considerando o comportamento humano antes, durante e depois da ocorrência de um evento" (Mattedi e Butzke, 2001, p. 7).

A abordagem sociológica considera os fatores sociais dos desastres, ou seja, o contexto social, a população atingida e o impacto sobre ela. Os estudos se concentram em três áreas: 1) o agente deflagrador do desastre; 2) o agente receptor do impacto; e 3) a resposta dada ao mesmo. A compreensão disso encerra três paradoxos de desenvolvimento disciplinar desta abordagem: 
a) o paradoxo da informação: os desastres, embora produzidos pela sociedade, são pouco conhecidos por ela; b) o paradoxo da intensificação: a área de estudos se torna menos interessante e menor, na medida em que aumenta o número de pesquisadores; e c) o paradoxo de gestão: os desastres são mais destrutivos na medida em que os dispositivos de gestão se tornam mais sofisticados. Mas que fatores causam os desastres? Fatores externos ou internos à sociedade? Duas correntes teórico-metodológicas predominam na resposta: o paradigma da ameaça externa e o paradigma da vulnerabilidade interna.

O paradigma da ameaça externa preocupa-se com os aspectos físicos e as características do evento-percepção. Estuda como fatores geofísicos podem se tornar ameaças para os indivíduos a elas expostos, qual a percepção e como respondem e se ajustam a elas (Montz e Tobin, 2011). Nessa abordagem, importa o fator deflagrador do evento e aspectos como velocidade, duração e extensão da destruição, aspectos secundários (sua presença ou não), o grau de preparação da população e a percepção que a mesma tem do risco (Cvetkovich e Earle, 1985). Uma vez que os processos meteorológicos se diferenciam, os hazards são classificados pelos seus mecanismos físicos (magnitude, duração e extensão espacial), pela distribuição temporal (frequência, sazonalidade e parâmetros diurnos) e espacial (localização geográfica) e, por fim, pela dinâmica de eclosão do evento (tempo do início, preparação e término) (Tobin e Montz, 1997).

O paradigma da vulnerabilidade interna foca nos aspectos sociais dos desastres por meio da relação impacto-organização. Essa abordagem entende que os desastres variam mais social do que geofisicamente e que o impacto na organização social determina a intensidade da destruição do evento. Considera as especificidades do contexto social conectando o cotidiano de sobrevivência da população aos eventos extremos que as impactam (Gilbert, 1995). Assim, o tipo de sociedade, os indivíduos e a posição que ocupam nela é que determinam a gravidade do desastre. Com o foco nas respostas, ganha relevância a organização social (Quarantelli, 1994). A organização social pré-impacto influencia o grau de vulnerabilidade aos desastres. Neste sentido, o agente do desastre é intrínseco ao contexto social e exprime a "materialização da vulnerabilidade social" (Pelanda, 1981), influenciando na escolha das estratégias de gestão dos desastres. 


\section{Estratégias de gestão dos desastres}

A história da gestão dos desastres passou por diversas fases. Uma privilegiava a preparação para as situações de emergência no pós-impacto do evento. Com a mudança de paradigmas, a fase pré-impacto passou a ter mais atenção. A GD tem sido a forma mais comumente adotada pelo Estado, que, progressivamente, foi se ocupando da questão. A intensificação da gestão da crise recebeu o protagonismo estatal que propiciou a centralidade do Estado e das políticas públicas. No entanto, a atuação do Estado na área é centralizadora. Uma pesquisa sobre a percepção e a gestão dos desastres em Blumenau constatou que a gestão está centralizada na defesa civil (DC). Percebe-se uma dicotomia entre as ações desta e o conhecimento acadêmico-científico, bem como a não consideração dos saberes locais na gestão (Avila, 2015). Constata-se, ainda, ausência de integração nos diferentes níveis de gestão, premissa de Narváez, Lavell e Ortega (2009) para uma GRD eficiente.

A história da GRD tem origem na década de 1990. Conceitualmente, surgiu para substituir as noções de GD existentes até então e que privilegiavam a preparação para o pós-impacto dos desastres. A mudança é estrutural, pois direciona o foco que antes privilegiava a fase pós-impacto - preparação e gestão dos desastres - para a fase pré-impacto - prevenção e mitigação do risco de desastres (Narváez, Lavell e Ortega, 2009). A GRD compreende que o risco é construído socialmente, ou seja, a sociedade tem participação direta na sua construção. Enquanto a GD concentrava sua atuação nas perdas e danos do pós-impacto, a GRD caracteriza-se pela presença do risco, ou seja, pela probabilidade de perdas e danos futuros à sociedade. É uma mudança substancial que redireciona a ação da gestão, perceptível nas diferenciações existentes entre as fases da GD e da GRD.

A GD possui duas fases distintas: 1) a pré-desastre, considerada a fase de redução do risco de desastres (RRD), caracterizada pela preparação, mitigação e prevenção; e 2) a pós-desastre ou a fase de GD, caracterizada pela resposta, recuperação e retomada do desenvolvimento (Asghar, Alahakoon e Churilov, 2011). Quanto aos modelos de GD, os mesmos podem ser classificados em duas categorias: o linear (clássico ou tradicional) e o circular (modelo de Kelly). O modelo linear (Figura 1) caracteriza-se por uma dicotomia, não considera o 
momento de ocorrência da crise (fase transdesastre), impedindo a vinculação das etapas do processo de gestão. Ou seja, privilegia a fase pré e pós-desastre e tem dificuldades em atuar com a integração de dados e a tomada de decisão que, geralmente, é centralizada (Albtoush, Dobrescu e Ionescou, 2011).

Figura 1. Modelo linear ou clássico (tradicional) de gestão de desastres

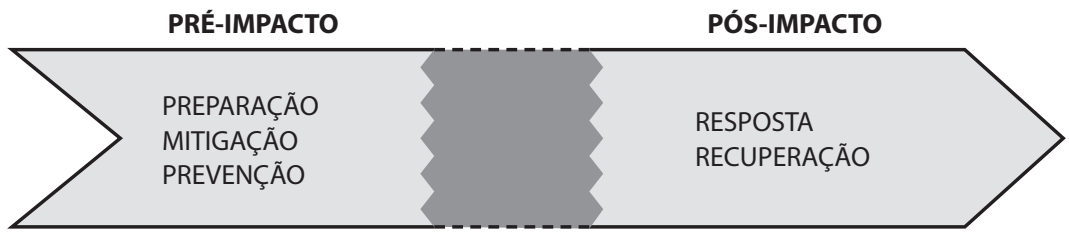

Fonte: Elaborado pelos autores (2016).

O modelo circular (Figura 2) considera o momento de crise (fase trans) e permite compreender os desastres como um processo integrado. O mesmo requer o desenvolvimento de um banco de dados abrangente com informações do impacto, da entrada e saída da situação de crise. Também requer treinamento específico para os atores sociais que trabalharão com as informações e exige uma infraestrutura de alta tecnologia para alcançar resultados razoáveis.

Figura 2. Modelo circular de gestão

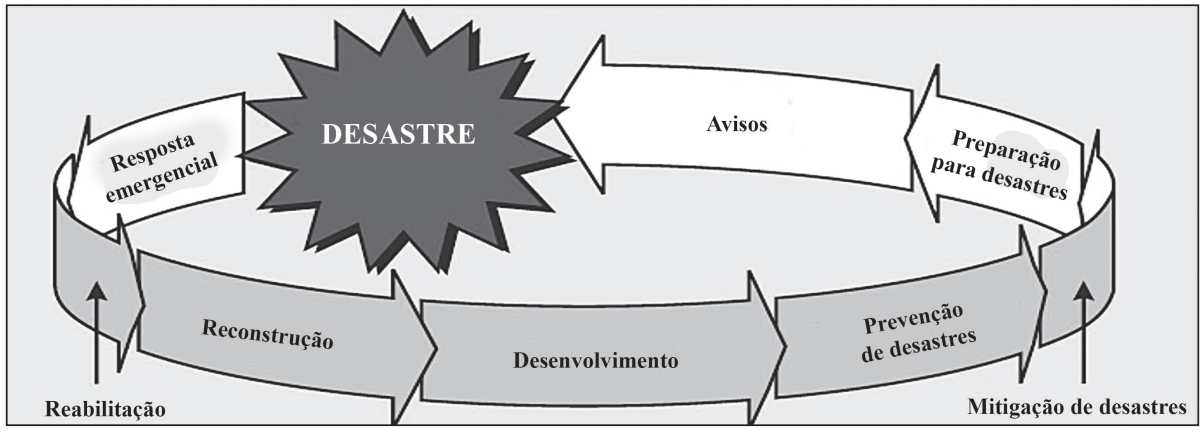

Fonte: Albtoush, Dobrescu e Ionescu (2011). 
Um modelo de GD possui quatro fases: hazard, gestão do risco (GR), mitigação e preparação. Sua tarefa é avaliar o perigo ou ameaça (hazard) e oferecer informações para que a fase de GR possa ser realizada com eficácia (Asghar, Alahakoon e Churilov, 2011; Manitoba, 2002). No entanto, não consegue estabelecer integração entre as fases, elementos importantes no processo de GD (Idem). Possui algumas características semelhantes aos modelos integrado, global e sistema hierárquico de gerenciamento de emergências (HSEM, na sigla em inglês), considerados modelos de GRD que possuem três fases: 1) identificação do risco - percepção do risco e definição de vulnerabilidades e ameaças; 2) redução do risco — prevenção e mitigação do risco; e 3) gestão do desastre, da qual fazem parte a resposta dada (resposta imediata) no pós-impacto, a reabilitação e a reconstrução ou retomada do desenvolvimento (Narváez, Lavell e Ortega, 2009; Arce e Córdoba, 2012).

A GRD é um "proceso social cuyo fin último es la previsión, la reducción y el control permanente de los factores de riesgo de desastre en la sociedad, en consonancia con, e integrada al logro de pautas de desarrollo humano, económico, ambiental y territorial, sostenibles" (Narváez, Lavell e Ortega, 2009 , p. 33). Esse controle, via políticas e estratégias de ação, se dá nos níveis de gestão territoriais (global, regional, local, comunitário e familiar) e setoriais (federal, estadual, municipal e internacional). É tipificada por um sistema integrado de gestão composto de seis processos: "1) generar conocimiento sobre el riesgo de desastres en sus diferentes ámbitos, 2) prevenir el riesgo futuro, 3) reducir el riesgo existente, 4) preparar la respuesta, 5) responder y rehabilitar, y 6) recuperar y reconstruir" (Idem, p. 62). Esses processos são contínuos, interdependentes, completos e imersos na dinâmica do desenvolvimento.

O modelo global foi construído a partir de duas premissas e a integração entre as mesmas: 1) avaliação do risco e de GR; 2) ações de GR e de GD (Asghar, Alahakoon e Churilov, 2011). Essas premissas configuram-se nas fases pré e pós-impacto dos desastres e a articulação entre elas. Pode-se dizer que esse modelo considera a fase transdesastre, ou seja, o momento de ocorrência do fenômeno. Já o HSEM (Albtoush, Dobrescu e Ronescou, 2011) introduz uma melhoria em relação aos modelos existentes até então. Possui seis componentes 
principais, o que o identifica muito com os seis processos do modelo integrado: planejamento estratégico, avaliação de risco e perigo, GR, GD, monitoramento e avaliação. Como o modelo global, o HSEM se divide em duas fases: a avaliação e a GR e a relação entre a GR e a GD (Idem).

Esses modelos podem ser considerados de GRD, pois preenchem as lacunas existentes dos modelos atuais. Ou seja, abrangem as diferentes fases de um desastre (pós, trans e pós-impacto do evento) e têm capacidade de lidar com situações complexas e difíceis que ocorrem durante e após as situações de emergência. Conforme Albtoush, Dobrescu e Ronescou (2011), um modelo de GRD precisa trabalhar no nível multidimensional e multidisciplinar. Deve ser dinâmico e capaz de manter a multi-interdependência entre os eventos, ações, atores, contextos e outros fatores envolvidos no processo de gestão. Partindo do caráter multidimensional, multidirecional, multissignificativo (Mattedi, 2015) e multi-interdependente dos desastres, desenhamos uma proposta de modelo de GRD (Figura 3), a qual denominamos modelo multidimensional de gestão de risco de desastres (MMGRD).

Essa proposta explicita os seis processos do modelo integrado de Narváez, Lavell e Ortega (2009). Considera os eventos, os atores e a interdependência entre as dimensões gestoras, suas ações e seu processo de continuidade e não linearidade de gestão. Também contempla o dinamismo e o caráter muldimensional e multidisciplinar citado por Albtoush, Dobrescu e Ionescou (2011) para um modelo ideal de GRD. Além dessas premissas, entendemos que um modelo de GRD ideal deve compreender também o caráter inter e transdisciplinar da gestão (Pineau, 2010), bem como considerar os saberes locais e a organização comunitária no processo gestor. O Serviço Social, por atuar diretamente com a população impactada e vulnerabilizada pelos desastres, pode se utilizar desse modelo. A profissão necessita aproximar o debate das questões ambientais e da gestão dos desastres, tanto na área de atuação profissional como na de formação, pesquisa e produção científica. 
Figura 3. Modelo multidimensional de gestão de risco de desastres

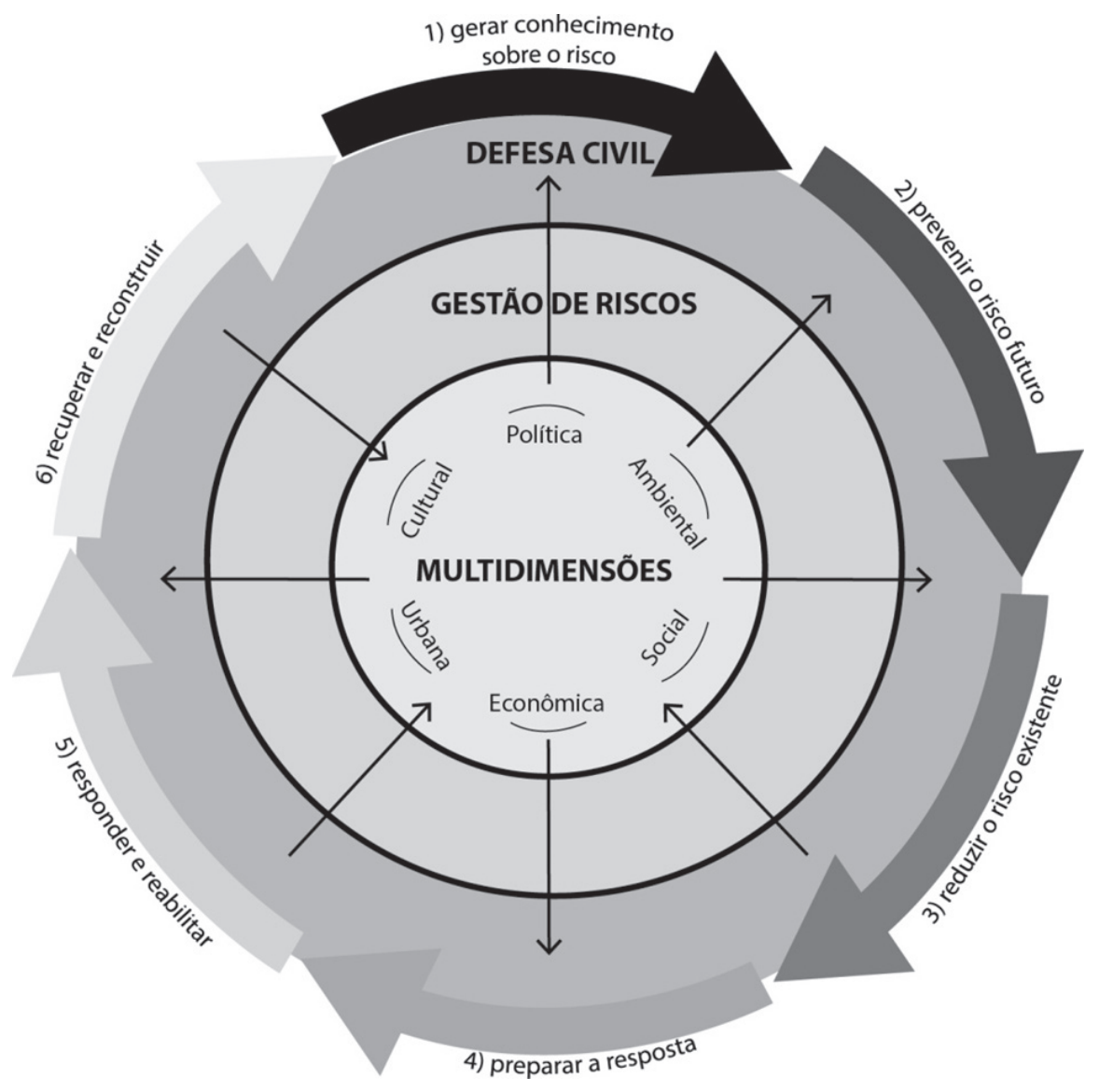

Fonte: Elaborado pelos autores (2016).

\section{Serviço Social e desastres}

Até 1990 “não existia na América Latina nenhum [...] trabalho consolidado e linha de pesquisa em instituições públicas que tivesse o ambiente como categoria importante para o conhecimento e intervenção prática do profissional 
em Serviço Social" (Loureiro, 2010, p. 18). A partir dessa década, o assunto passou a ter importância na agenda social da profissão, e referências ao tema passaram a ocorrer em artigos da área (Nunes, 2013). Nesse sentido, o Código de Ética do(a) Assistente Social, art. $3^{\circ}$, cita como dever profissional "participar de programas de socorro à população em situação de calamidade pública, no atendimento e defesa de seus interesses e necessidades" (CFESS, 1993). Em 2016, o Serviço Social completou oitenta anos no Brasil. A profissão nasceu no bojo do ideário católico de assistencialismo e caridade com um caráter extremamente conservador (Martinelli, 2009). Na década de 1940, a profissão passou por uma reorientação, e na década de 1960 ,

A profissão [...] direciona seus questionamentos ao Serviço Social tradicional através de um amplo movimento, de um processo de revisão global, em diferentes níveis: teórico, metodológico, operativo e político [...]. É no bojo deste movimento, de questionamentos à profissão [...] que a interlocução com o marxismo vai configurar para o Serviço Social latino-americano a apropriação de outra matriz teórica: a teoria social de Marx. (Yazbek, 2009, p. 147)

Essa fase de avaliação e transição da profissão, de um caráter conservador e tradicional para uma teoria social crítica, é denominada Movimento de Reconceituação.

Dentre os espaços ocupacionais da profissão situa-se a política pública de Assistência Social, coordenada pelo Ministério do Desenvolvimento Social e Combate à Fome (MDS) e que foi instituída a partir da Lei n. 8.742, de 7/12/1993, Lei Orgânica da Assistência Social (Loas). Em 2004, o Conselho Nacional de Assistência Social (CNAS) aprovou a Política Nacional de Assistência Social (PNAS), estabelecendo seus princípios, diretrizes, benefícios, serviços, entre outros (Brasil, 2009). E em 2005, o CNAS aprovou o Sistema Único de Assistência Social (Suas), organizando a política em níveis de complexidade, assim definidos: Proteção Social Básica e Proteção Social Especial de Média e Alta Complexidade (Resolução n. 130, de 15/7/2005). Com a tipificação dos serviços socioassistenciais (Resolução n. 109, de 11/11/2009), foram definidos os serviços que compõem cada nível do Suas (Quadro 1). 
Quadro 1. Organização dos serviços socioassistenciais da Assistência Social

\begin{tabular}{|l|l|}
\hline \multicolumn{1}{|c|}{ NÍVEIS DE COMPLEXIDADE } & \multicolumn{1}{c|}{ SERVIçoS SOCIOASSISTENCIAIS } \\
\hline I - Serviços de Proteção Social & a) Serviço de Proteção e Atendimento Integral à Família (Paif) \\
Básica & b) Serviço de Convivência e Fortalecimento de Vínculos \\
& c) Serviço de Proteção Social Básica no domicílio para pessoas com \\
& deficiência e idosos \\
\hline II - Serviços de Proteção Social & a) Serviço de Proteção e Atendimento Especializado a Famílias e Indivíduos \\
Especial de Média Complexidade & b) Serviço Especializado em Abordagem Social \\
& c) Serviço de Proteção Social a Adolescentes em Cumprimento de Medida \\
& $\begin{array}{l}\text { Socioeducativa de Liberdade Assistida (LA), e de Prestação de Serviços à } \\
\text { Comunidade (PSC) }\end{array}$ \\
& d) Serviço de Proteção Social Especial para Pessoas com Deficiência, Idosas \\
& e Famílias \\
\hline III - Serviços de Proteção Social & a) Serviço Especializado para Pessoas em Situação de Rua de Acolhimento Institucional nas seguintes modalidades: abrigo \\
Especial de Alta Complexidade & institucional, casa lar, casa de passagem, residência inclusiva \\
& b) Serviço de Acolhimento em República \\
& c) Serviço de Proteção em Situação de Calamidades Públicas e \\
& Emergências. \\
\hline
\end{tabular}

Fonte: Elaborado pelos autores (2016), adaptado de Brasil (2009), grifos nossos.

Dentre os Serviços de Proteção Social Especial de Alta Complexidade, situa-se o Serviço de Proteção em Situação de Calamidades Públicas e Emergências, o qual prevê a atuação dos trabalhadores da política de assistência social, dentre eles o(a) assistente social. Até a década de 1990, a categoria era vista apenas como executora de políticas sociais e suas atuações entendidas como ações pedagógicas e educativas. Essa visão ainda existe. No entanto, o(a) assistente social é profissional competente para atuar tanto na execução de políticas sociais, como na área de planejamento, formulação e avaliação de programas, projetos e gestão dessas políticas (Yazbek, 2009). Por isso, tem autonomia para contribuir no planejamento e administração de políticas de gestão dos desastres, bem como na pesquisa e produção científica na área. No entanto, o debate sobre os desastres não ocupa destaque no âmbito das entidades da categoria.

Os resultados de uma pesquisa realizada entre 17 e 26 de julho de 2016 comprovam essa afirmação. A pesquisa foi realizada nos sites dos 26 Conselhos Regionais de Serviço Social (Cress), no portal da Capes, que trata dos programas de pós-graduação da área, na Biblioteca Digital Brasileira de Teses e Disserta- 
ções (BDTD), no Banco de Teses e Dissertações do Portal da Capes (também na área do Serviço Social desse portal) e nos sites de divulgação dos três últimos Encontros de Pesquisadoras(es) do Serviço Social (Enpess). A metodologia de pesquisa nos sites das 26 regiões dos Cress consistiu em buscar a existência de grupos de trabalho (GTs) ou comissões temáticas (CTs) que discutem meio ambiente ou desastres. Após acesso aos sites dos conselhos, verificada a existência dos GTs e CTs, foram consultadas as ementas ou informações constantes.

No mesmo período, estendemos a pesquisa para a área de formação da profissão e constatamos, conforme o Documento de Área 2013 da Capes, que avaliou os programas de pós-graduação, que no Brasil há 31 programas de pós-graduação em Serviço Social (Capes, 2013). Realizamos buscas em 28 deles com o objetivo de verificar a existência de linha de pesquisa que contemple a discussão sobre desastres e meio ambiente. A mesma metodologia foi aplicada na área da produção científica. Realizamos buscas por títulos que contivessem as palavras meio ambiente ou desastre no site da BDTD e no Banco de Teses e Dissertações do Portal da Capes. No site da Capes, refinamos a pesquisa para a área do Serviço Social. Pesquisamos ainda nos sites de divulgação dos últimos encontros de pesquisadores do Serviço Social (Enpess) em 2012, 2014 e 2016. A Figura 4 e o Quadro 2 apresentam os resultados das pesquisas efetuadas.

Apenas a $7^{\mathrm{a}}$ Região do Cress (RJ) possui comissão que discute desastres. A $5^{\mathrm{a}}$ Região (BA) e $6^{\mathrm{a}}$ Região (MG) possuem comissões que discutem meio ambiente, muito embora a comissão de meio ambiente da $6^{\text {a }}$ Região trate apenas da coleta seletiva do lixo. Algumas regiões possuem GT que discute a questão urbana, como a $10^{\mathrm{a}}$ Região, ou o direito à cidade, como $11^{\mathrm{a}}$ Região, mas nas ementas das mesmas nada constava sobre desastres ou meio ambiente.

Na pós-graduação em Serviço Social, apenas três programas possuem linha de pesquisa abordando meio ambiente ou desastres. Na BDTD, ao digitarmos desastre + Serviço Social, encontramos dezoito documentos, doze com a palavra desastre no título. No Banco de Teses e Dissertações da Capes, digitamos desastre na busca on-line e encontramos 333 documentos. Ao pesquisar quais deles continham as palavras vulnerabilidade e desastre no título, encontramos 29 documentos para vulnerabilidadee 155 para desastre. Na área de Serviço 
Figura 4. Meio Ambiente e desastres nas regiões dos Cress no Brasil

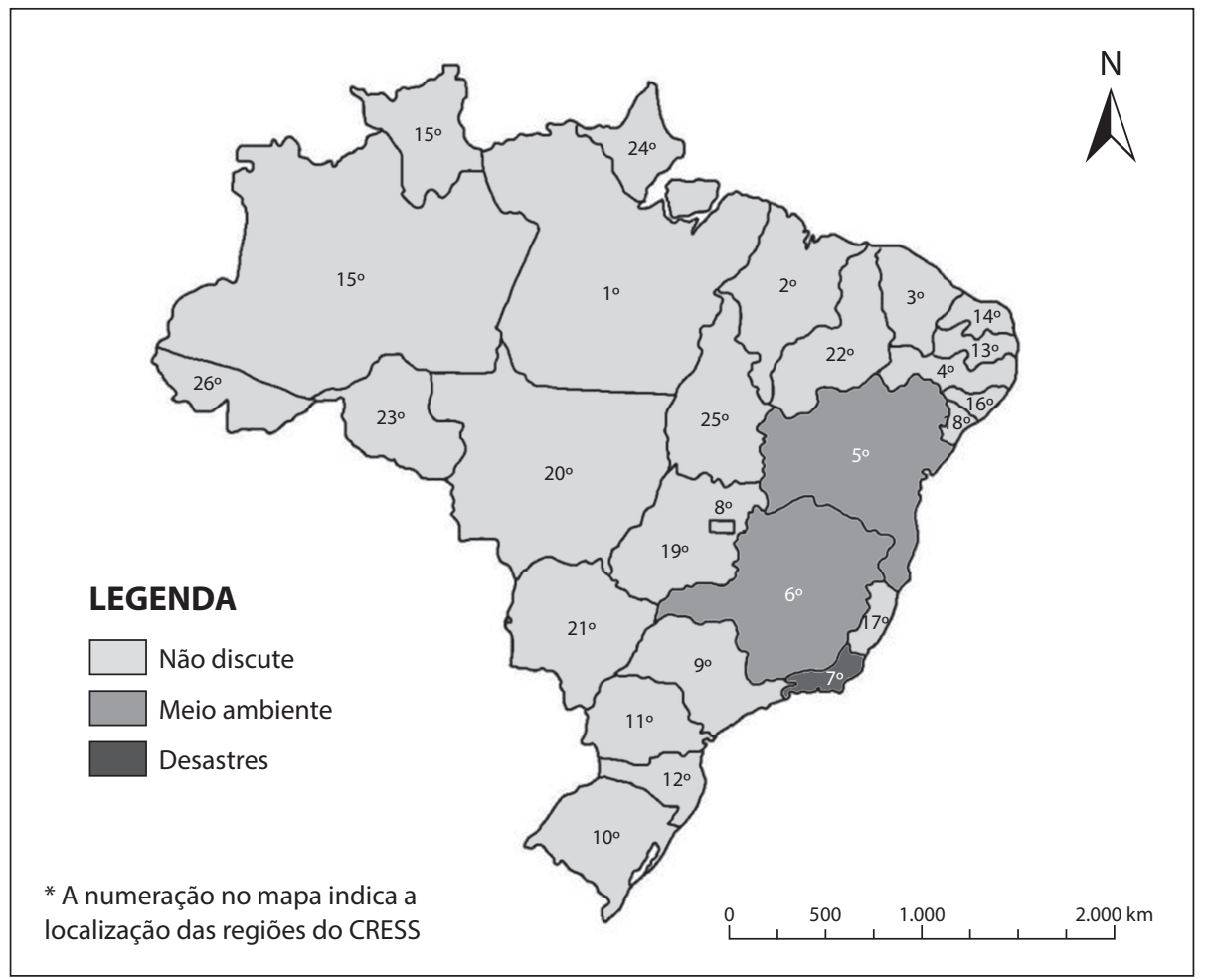

Fonte: Elaborado pelos autores (2016) a partir de dados dos sites das regiões do Cress.

Social da Capes, pesquisamos 2 mil documentos. Encontramos três com a palavra desastre e três com a palavra vulnerabilidade no título (não se tratava de vulnerabilidade a desastres), e 25 com a temática da questão ambiental. Nos dados dos Enpess (2012, 2013 e 2016), verificamos que a questão ambiental já é discutida nos Grupos de Trabalho e Pesquisa (GTPs). No entanto, os desastres não possuem eixo temático específico.

A pesquisa realizada comprova que a discussão teórica e a produção científica acadêmica sobre desastres ainda é incipiente no Serviço Social. Embora a profissão atue na emergência dos desastres e discuta a questão ambiental, esse 
Quadro 2. Serviço Social e desastres

\begin{tabular}{|c|c|}
\hline LOCAIS DE BUSCA & RESULTADOS \\
\hline UNIVERSIDADES & PÓS-GRADUAÇÃO EM SERVIÇO SOCIAL \\
\hline $\begin{array}{c}\text { UFSC - PUCSP - UFRGS } \\
\text { - UFRJ - UFPB - PUCRJ } \\
\text { UFPE - UNB - Unesp - UEL } \\
\text { - Ufes - UFPA - UFRN } \\
\text { - Ufal - UFJ - Ucsal } \\
\text { - Ucpel - PUCGO - Ufam } \\
\text { — UFMT - UFV - Unicsul } \\
\text { - Emescan - UFCE - Uece } \\
\text { - Fufse - } \\
\text { UFF (Política Social) } \\
\text { UFF (Serviço Social e } \\
\text { Desenvolvimento Regional). }\end{array}$ & $\begin{array}{l}1 \text { - O Programa (M + D) da Escola de Serviço Social da UFRJ possui uma } \\
\text { linha de pesquisa denominada "Temas urbanos". Discute meio ambiente. } \\
2 \text { - O Programa de Pós-Graduação em Serviço Social (M + D) da PUCRJ } \\
\text { possui uma linha de pesquisa denominada "Questões socioambientais, urbanas } \\
\text { e formas de resistência social". } \\
3 \text { - O Programa de Pós-Graduação em Serviço Social da UFPE possui uma } \\
\text { linha de pesquisa denominada "Capitalismo contemporâneo, questão ambiental } \\
\text { e Serviço Social", que aborda a questão ambiental, focalizando a ação do } \\
\text { Serviço Social nesse espaço. }\end{array}$ \\
\hline BDTD & $\begin{array}{l}\text { BIBLIOTECA DIGITAL BRASILEIRA DE TESES E DISSERTAÇÕES } \\
\text { http://bdtd.ibict.br/vufind/ }\end{array}$ \\
\hline Desastres + Serviço Social & $\begin{array}{l}\text { Encontrados dezoito documentos (entre teses e dissertações). } \\
\text { Pelos títulos, doze tratavam do tema desastres. }\end{array}$ \\
\hline CAPES & $\begin{array}{l}\text { BANCO DE TESES E DISSERTAÇÕES DO PORTAL DA CAPES } \\
\text { http://bancodeteses.capes.gov.br/banco-teses/\#/ }\end{array}$ \\
\hline $\begin{array}{c}\text { Palavra desastre nos documentos } \\
\text { em geral } \\
\text { Palavras desastre e } \\
\text { vulnerabilidade no título }\end{array}$ & $\begin{array}{l}\text { - } 333 \text { documentos (teses e dissertações). } \\
\text { - } 333 \text { documentos (da primeira pesquisa) } \\
\text { Vulnerabilidade }-29 \text { documentos } \\
\text { Desastre }-155 \text { documentos }\end{array}$ \\
\hline CAPES - SSO & $\begin{array}{l}\text { BANCO DE TESES E DISSERTAÇÕES DA ÁREA DE SERVIÇO } \\
\text { SOCIAL - PORTAL DA CAPES }\end{array}$ \\
\hline $\begin{array}{l}\text { Pesquisa por títulos em } 2000 \\
\text { documentos da área }\end{array}$ & $\begin{array}{l}\text { - Três documentos (dissertações) com a palavra desastre no título. } \\
\text { - Três com a palavra vulnerabilidade (não se tratava de vulnerabilidade a } \\
\text { desastres). } \\
\text { - } 25 \text { documentos com as palavras questão ambiental, socioambiental, meio } \\
\text { ambiente e educação ambiental nos títulos }\end{array}$ \\
\hline EVENTOS & $\begin{array}{c}\text { ENCONTRO NACIONAL DE PESQUISADORAS(ES) } \\
\text { DO SERVIÇO SOCIAL } \\
\text { http://ulbra-to.br/cursos/Servico-Social/noticia/2012/09/19/XIII-ENPESS-2012 } \\
\text { http://fescemp.com.br/index.php/typography/cpa/item/42-xiv-encontro- } \\
\text { nacional-de-pesquisadores-em-servico-social-enpess } \\
\text { http://www.abepss.org.br/enpess/trabalhos-29 }\end{array}$ \\
\hline ENPESS & $\begin{array}{l}\text { Pesquisamos os grupos temáticos de pesquisa (GTP) em sites de divulgação do } \\
\text { XV Enpess ( } 4 \text { a 9/11/2016 em Ribeirão Preto/MG) } \\
\text { XIV Enpess ( } 30 / 11 \text { a 4/12/2014, realizado em Natal/RN) } \\
\text { XIII Enpess ( } 5 \text { a 9/11/2012, realizado em Juiz de Fora/MG) } \\
\text { Os três eventos possuíam um GTP denominado "Questão agrária, urbana, } \\
\text { ambiental e o Serviço Social". }\end{array}$ \\
\hline
\end{tabular}

Fonte: Elaboração dos autores (2016) a partir dos sites das categorias pesquisadas. 
debate precisa ser ampliado para a temática da vulnerabilidade e gestão dos desastres. A inserção dessas temáticas pode ocorrer em dois campos: da políti$c a$ e da formação. Portanto, o Serviço Social, tanto por meio da formação de seus profissionais como por meio da atuação profissional, tem condições de fornecer subsídios para a gestão dos desastres. Essa, por sua vez, pode intervir diretamente na dinâmica de formação da profissão (Figura 5), numa relação de interdependência a partir da inserção da vulnerabilidade e gestão dos desastres na agenda da categoria.

Figura 5. Dinâmica de formação e atuação profissional

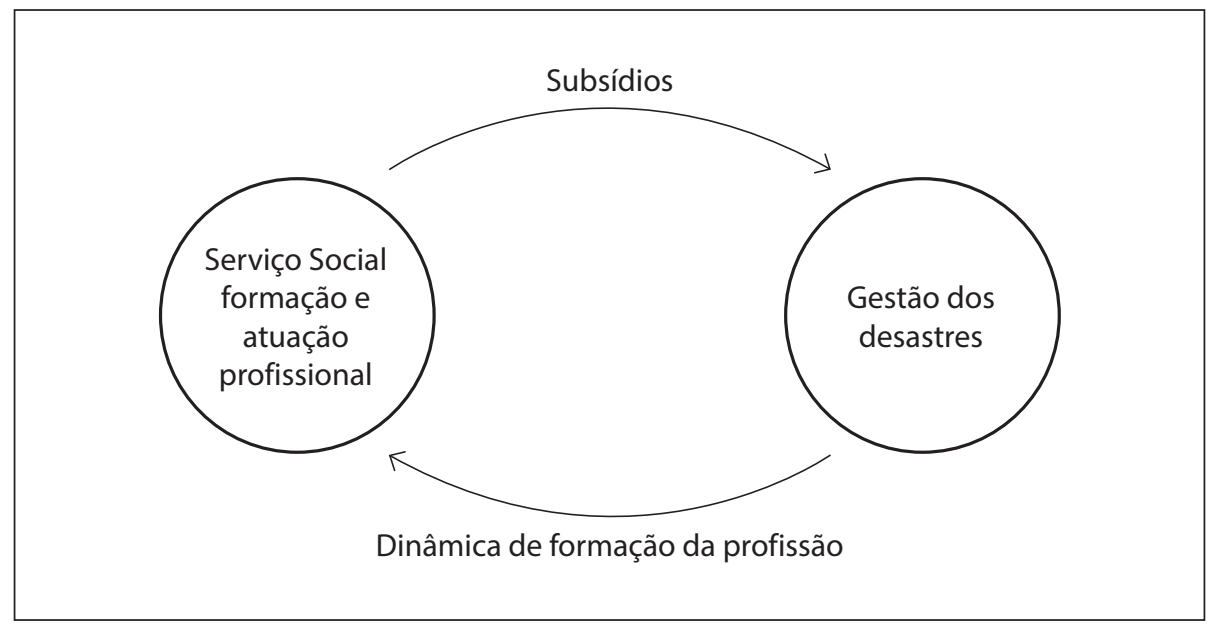

Fonte: Elaborado pelos autores (2016).

Para Nunes (2013, p. 207),

o assistente social poderá se inserir neste campo de forma propositiva, crítica, com ações sustentadas nos eixos teórico-metodológico, ético-político e técnico-operativo, onde o projeto ético-político do Serviço Social constitui ferramenta essencial e referência a todos os profissionais que buscam imprimir um diferencial de qualidade em seu exercício profissional. 
Segundo a autora, a forma como o Serviço Social (e outras disciplinas das Ciências Sociais) compreendem os desastres é fundamental. Conforme afirma,

tem-se como pressuposto que apreender os desastres como um processo sócio [sic] histórico decorrente, sobretudo, do padrão de desenvolvimento em curso, é fundamental para potencializar novos paradigmas para a prevenção de desastres, o que repercutirá na elaboração e implementação de políticas públicas. Afirma-se, ainda, a relevância das ciências sociais nas respostas às questões socioambientais e na prevenção e atenção aos desastres, na medida em que assumam um enfoque integral, compreendendo as complexas relações entre sociedade e natureza, avançando na construção de uma proposta interdisciplinar, a partir de uma visão crítica dos marcos conceituais e que a prática da interdisciplinaridade potencializa. (Nunes, 2013, p. 208)

Diante do exposto, concluímos ser necessária a construção de novos paradigmas no trato das questões socioambientais na área do Serviço Social. Evidenciamos a necessidade urgente da inclusão desse campo de estudos e pesquisa na área: a inserção da vulnerabilidade e gestão dos desastres na agenda social da categoria.

\section{Considerações finais}

A relação entre Serviço Social e desastres está, muitas vezes, restrita ao campo da intervenção do profissional junto à população atingida. No entanto, além de atuar na mitigação das necessidades imediatas da população (gestão da crise e acesso aos serviços básicos — abrigo, alimentação, vestuário), o(a) assistente social tem capacidade operativa para atuar na articulação entre as redes de apoio social (saúde, habitação, defesa civil etc.). A prática interorganizacional da profissão é imprescindível no momento do desastre e no pós-impacto deste, porque essas redes são quase sempre interrompidas em situações de crise e precisam ser restabelecidas rápida e eficientemente (Zakour, 2007 e 2008). No entanto, a fase pré-impacto dos desastres também é fundamental. Por isso a importância de articular o conhecimento, vivenciado 
na prática, sobre desastres à dinâmica de formação da profissão (pesquisa e produção científica).

Buscamos discutir a inserção da vulnerabilidade e da gestão dos desastres na agenda do Serviço Social. Apesar de não ser possível o aprofundamento em todas as temáticas abordadas, o trabalho discutiu a centralidade da atuação da profissão no atendimento das situações de emergência. Embora essa atuação já ocorra, verifica-se que a mesma se restringe às fases trans (momento do desastre) e pós-impacto dos eventos. Esta precisa ser ampliada para as fases pré-impacto de desastre, principalmente no planejamento e na implementação de políticas públicas para a área. No entanto, para além da prática interventiva do(a) assistente social, a categoria é chamada a dar sua contribuição no campo teórico e de formação da profissão. Para tanto, é preciso tornar os desastres um campo de estudos do Serviço Social, tanto em nível de graduação como de pós-graduação.

Recebido em 3/10/2016 - Aprovado em 14/2/2017

\section{Referências bibliográficas}

ALBTOUSH, R.; DOBRESCU, R.; IONESCOU, F. A hierarchical model for emergency management systems. U.P.B. Sci. Bull., Series C, v. 73, n. 2, p. 53-62, 2011. Disponível em: <http://www.scientificbulletin.upb.ro/rev_docs/arhiva/full87242.pdf >. Acesso em: 21 mar. 2016.

ARCE, M. F.; CÓRDOBA, A. C. Las TIC y la gestión del riesgo a desastres. In: UNESCO. Hacia la Sociedad de la Información y el Conocimiento, 2012. p. 257-282.

ASGHAR, S.; ALAHAKOON, D.; CHURILOV, Leonid. A Comprehensive Conceptual Model for Disaster Management. Clayton School of Information Technology, Monash University, Austrália, 2011. Disponível em: <http://sites.tufts.edu/jha/files/2011/04/ a193.pdf >. Acesso em: 28 mar. 2016.

AVILA, M. R. R. Em Blumenau tudo é risco: a percepção e a gestão dos desastres baseado no estudo de caso da Comunidade da Rua Pedro Krauss Sênior (Blumenau/SC) no 
período de 2008 a 2015. 216 p. Dissertação (Mestrado) — Programa de Pós-Graduação em Desenvolvimento Regional. Universidade Regional de Blumenau, Blumenau, 2015.

AYSAN, Y. “Os pisos no âmbito do vulnerável": redução de desastres como uma estratégia para reduzir a pobreza. Apresentado no Banco Mundial, Grupo Consultivo para a Redução de Desastres Globais. Reunião: 1-2/6/1999, Paris. Disponível em: $<$ http://www.radixonline.org/humanrights2.htm>. Acesso em: 11 fev. 2016.

BRASIL. Tipificação Nacional de Serviços Socioassistenciais. Texto da Resolução n. 109 de 11 nov. 2009, publicada no Diário Oficial da União em 25 nov. 2009, Brasília.

CAPES. Coordenação de Aperfeiçoamento de Pessoal de Nível Superior. Documento de Área 2013. Área de Avaliação: Serviço Social. Diretoria de Avaliação. Capes, 2013. Disponível em: <https://www.capes.gov.br/images/stories/download/avaliacaotrienal/ Docs_de_area/Servi\%C3\%A7o_Social_doc_area_e_comiss\%C3\%A3o_16out.pdf $>$. Acesso em: 18 jul. 2016.

CARR, L. J. Disaster and the Sequence-Pattern Concept of Social Change. American Journal of Sociology, V. 38, n. 2, Sep., p. 207-218, 1932.

CEPED. Atlas brasileiro de desastres naturais (1991 a 2010). Florianópolis, 2013. v. Brasil.

CFESS. Resolução CFESS n. 273/93. Institui o Código de Ética Profissional dos Assistentes Sociais. Brasília: CFESS, 1993.

CVETKOVICH, G.; EARLE, T. C. Classifying hazardours events. Journal of Environmental Psychology, v. 5, p. 5-35, 1985.

D. GUHA-SAPIR; BELOW, R.; HOYOIS, P. The OFDA/CRED International Disaster. Database. Disponível em: $<$ http://www.emdat.be/disaster_trends/index.html $>$. Acesso em: 1 mar. 2016.

FREIRIA, S. Título da página electrónica: EM-DAT, The International Disaster Database - Centre for Research on the Epidemiology of Disaster (CRED). Revista Critica de Ciências Sociais, Coimbra, n. 93. p. 208-209, 2011. Disponível em: $<$ https://rccs.revues. org/1416>. Acesso em: 1 ago. 2016.

GILBERT, C. Studying disaster: a review of the main conceptual tools. International Journal of Mass Emergencies and Disasters, v. 13, n. 3, p. 231-240, 1995. 
HEWITT, K. Regions of risk: a geographical introduction to disasters - themes in resource management. Goodreads, EUA, 1997. Disponível em: < http://translate.google. com.br/translate?hl=ptBR\&sl=en\&u=http:/www.goodreads.com/author/show/1573966. Kenneth_Hewitt\&prev=/search\%3Fq\%3Dkenneth\%2Bhewitt\%26hl\%3DptBR\%26rlz\%3D1T4GGNI_pt-BRBR550BR550>. Acesso em: 15 jun. 2016.

HEWITT, K. Gifts and perils of landslides: catastrophic rockslides and related landscape developments are an integral part of human settlement along upper Indus streams. American Scientist, set./out. 2010. Disponível em: <http://ranslate.google.com.br/ translate?hl=ptBR\&sl=en\&u=http://www.americanscientist.org/authors/detail/ kennethhewitt\&prev $=/$ search $\% 3 \mathrm{Fq} \% 3$ Dkenneth $\% 2$ Bhewitt $\% 26$ hl\%3 DptBR\%26rlz\%3D1T4GGNI_pt-BRBR550BR550>. Acesso em: 16 abr. 2016.

KATES, R. Gilbert White 1911-2006: a biographical memoir. Washington D. C.: National Academy of Sciences, 2011.

LOUREIRO, C. F. B. Prefácio. In: SILVA, M. G. Questão ambiental e desenvolvimento sustentável: um desafio ético-político ao Serviço Social. São Paulo: Cortez, 2010.

MANITOBA Health Disaster Management. Disaster Management Model for the Health Sector: Guideline for Program Development. Version 1, nov. 2002. Disponível em: $<$ http://www.gov.mb.ca/health/odm/model>. Acesso em: 28 mar. 2016.

MARCHEZINI, V. Desafios de gestão de abrigos temporários: uma análise sociológica de inseguranças e riscos no cotidiano de famílias abrigadas. São Carlos: UFSCar, 2010.

MARTINELLI, M. L. Serviço Social: identidade e alienação. 13. ed. São Paulo: Cortez, 2009.

MATTEDI, M. A. Pensando com o desenvolvimento regional: subsídios para um programa forte em desenvolvimento regional. Revista Brasileira de Desenvolvimento Regional - RBDR, Blumenau, v. 2, n. 2, p. 59-105, 2015.

; BUTZKE, I. C. A relação entre o social e o natural nas abordagens de hazards e de desastres. Ambiente \& Sociedade, São Paulo, n. 9, p. 10-21, 2001.

MONTZ, B. E.; TOBIN, G. A. Natural hazards: na evolving tradition in applied geography. Applied Geography, v. 31, p. 1-4. 2011.

NARVÁEZ, L.; LAVELL, A.; ORTEGA, G. P. La gestión del riesgo de desastres: un enfoque basado en procesos. Proyecto Apoyo a la Prevención de Desastres en la Comunidad Andina — Predecan. 1. ed., Lima, 2009. 106 p. 
NUNES, L. S. A questão socioambiental e a atuação do assistente social. Textos \& Contextos, Porto Alegre, v. 12, n. 1, p. 196-212, jan./jun. 2013. Disponível em: <http:// revistaseletronicas.pucrs.br/ojs/index.php/fass/article/viewFile/13337/9623>. Acesso em: 14 mar. 2016.

; SILVA, A. G. de M. O debate em torno da questão socioambiental na formação profissional do assistente social. In: CONGRESSO CATARINENSE DE ASSISTENTES SOCIAIS, 22 a 24 de agosto de 2013, Florianópolis, SC.

ONU. Informe de evaluación global sobre la reducción del riesgo de desastres 2015 (GAR15). UNISDR, 2015.

PELANDA, C. Disaster and sociosystemic vulnerability. Newark: Preliminary Paper \#68 — Disaster Research Center/University of Delaware, 1981.

PERRY, R. W.; QUARANTELLI, E. L. What is a disasters? New answers to old questions. Bloomington: Xlibris, 2005.

PHILLIPS, B. D.; et al. Social vulnerability to disasters. CRC Press, 2010.

PINEAU, G. Estratégia universitária para a transdisciplinaridade e a complexidade. Instituto Paulo Freire, 2010. Disponível em: <http://www.rizoma-freireano.org/index. php/estrategia-universitaria-para-a-transdisciplinaridade-e-a-complexidade--gastonpineau>.Acesso em: 9 maio 2016.

PRINCE, S. H. Catastrophe and social change: based upon a sociological study of the Halifax disaster. Doctoral dissertation. Nova York: Columbia University, Department of Political Science, 1920.

QUARANTELLI, E. L. Disasters: theory and research. Beverly Hülls: Sage, 1978.

. Disasters studies: the consequences of the historical use of a sociological approach in the development of research. International Journal of Mass Emergencies and Disasters, v. 12, n. 1, p. 25-49, 1994.

REVET, S. El mundo internacional de las catástrofes naturales. Política y Sociedad, Madri, v. 48, n. 3, p. 537-554, 2011.

SAMAGAIA, J.; ANGIONI, M. Situação dos desabrigados/atingidos pelo desastre e assistência (des)organizada. In: FRANK, B.; SEVEGNANI, L. (Orgs.). Desastre de 2008 no Vale do Itajaí: água, gente e política. Blumenau: Agência de Água do Vale do Itajaí, 2009. p. 140-147. 
SANTOS, R. dos. Gestão de desastres e política de assistência social. Revista Katálysis, Florianópolis, v. 15, n. 1, jan./jun. 2012.

SANTOS, T. S. O fazer profissional à luz dos pressupostos teórico-metodológicos. In: III SIMPÓSIO MINEIRO DE ASSISTENTES SOCIAIS, realizado de 7 a 9/6/2013. Cress, 8. Região. Belo Horizonte-MG.

TOBIN, G. A.; MONTZ, B. E. Natural hazards: explanation and integration. Nova York: Guilford Press, 1997.

UNISDR. United Nations Office for Disaster Risk Reduction. History. Disponível em: $<$ http://www.unisdr.org/who-we-are/history>. Acesso em: 20 dez. 2015.

YAZBEK, M. C. Fundamentos históricos e teórico-metodológicos do Serviço Social. In: CEFSS/ABEPSS. Serviço Social: direitos sociais e competências profissionais. Brasília: Cefss/Abepss, 2009. 760 p.

ZAKOUR, M. J. Social work and disasters. In: McENTIRE, D. A. (Ed.). Disciplines, disasters, and emergency management. The convergence and a divergence of concepts, issues and usual trends from the research literature. Springlief: C. C. Thomas, 2007. p. 124-141.

. Disasters research in social work. Journal of Social Service Research, 22:1-2, 7-25, New Orleans, 1997. Publicação em 25 set. 2008. Disponível em: <http://dx.doi. org/10.1300/J079v22n01_02>. Acesso em: 17 maio 2016. 